\title{
Scope and Limitations of a General Unknown Screening by Gas Chromatography-Mass Spectrometry in Acute Poisoning*
}

\author{
C. Köppel \\ Poison Information Center, Department of Nephrology and Intensive Care Medicine, Virchow-Klinikum, \\ Humboldt-Universität zu Berlin, Berlin, Germany
}

\author{
J. Tenczer \\ Department of Forensic Toxicology, Landesinstitut für Gerichtliche und Soziale Medizin, Berlin, Germany
}

\begin{abstract}
The gold standard for the diagnosis of acute poisoning is toxicological analysis. Because information on the incorporated toxic substance provided by the patient or his relatives is known from experience to be unreliable in about $40 \%$ of all intoxications, a screening procedure that covers most relevant drugs and toxicants is required rather than an analytical procedure optimized for the identification of a single class of substances. The special task for a general unknown screening procedure is to identify a toxic substance among endogenous or food-derived substances as well as environmental toxicants in a biological matrix on an emergency basis. Because the unknown toxic substance may vary considerably in its physicochemical properties and its concentration range, a universally applicable screening procedure is required. Although gas chromatography-mass spectrometry has been used for three decades, it still offers many unique advantages in terms of sensitivity, specificity, reliability, and coverage of a large number of toxic substances. Because the procedure has to be kept as simple and as short as possible, compromises have to be made with respect to extraction, derivatization, and mass-spectral techniques. The specimen of choice for a general unknown screening is - if available-urine. The standard mode of ionization is electron impact. The identification of unknown substances is highly challenging because, in our experience, previously unknown metabolites may be detected rather frequently in acute poisoning. Although an automated mass spectra library search considerably facilitates the identification process, expert knowledge on the identification of substances not included in the library as well as knowledge in clinical toxicology and metabolism is indispensable. (] Am Soc Mass Spectrom 1995, 6, 995-1003)
\end{abstract}

$\mathrm{I}$ n Germany, about 4000 to 5000 patients die from acute poisoning each year, which is about half the number of individuals that die in traffic accidents. An estimated 200,000 patients per year are treated for acute poisoning in emergency wards $[1,2]$. Table 1 gives an overview of the most frequently ingested drugs in suicidal intoxications. In Germany, about two thirds of acute poisonings are due to drug overdose. More than one drug is ingested in $46 \%$ of all cases of drug overdose. About two thirds of the intoxications are due to suicidal drug ingestion. Ethanol is involved in about $40 \%$ of all acute suicidal poisonings $[3,4]$.

The diagnosis of acute poisoning is based on the history, clinical symptomatology (which, however, is generally rather unspecific), standard laboratory tests,

Address reprint requests to Dr. C. Köppel, Poison Information Center, Department of Nephrology and Intensive Care Medicine, VirchowKlinikum, Humboldt-Universität zu Berlin, Augustenburger Platz 1, D-13353 Berlin, Germany.

* Dedicated to Professor Dr. F. W. McLafferty.

and toxicological analysis. There is general agreement that emergency toxicological analysis is the gold standard for the diagnosis of acute poisoning $[2,5]$. Unfortunately, only few intoxications are characterized by highly specific symptoms that allow diagnosis primarily on the basis of the clinical findings [4]. It has been demonstrated empirically that information on the ingested drug or toxicant obtained from patients or their relatives is incomplete or incorrect in about $40 \%$ of all poisonings [2]. Therefore, an emergency analytical procedure should not only aim at the most probable ingested toxic substance, but also should include all of the most commonly ingested drugs or toxicants. A general unknown screening procedure that covers as many drugs and toxicants as possible and that has a high reliability, sensitivity, and specificity is required [5].

A general unknown screening is a completely different task in comparison to identification and quantitation of a given substance in a biological matrix. In 
Table 1. Frequency, prescription, and elimination characteristics of drugs in drug overdose in patients admitted to the medical intensive care unit of the Universitätsklinikum Rudolf Virchow, Berlin, in 1993 ( $11=.323$ patients)

\begin{tabular}{lcccc}
\hline Drug & $\begin{array}{c}\text { Frequency } \\
(\%)\end{array}$ & $\begin{array}{c}\text { Under } \\
\text { prescription } \\
\text { in Germany }\end{array}$ & $\begin{array}{c}\text { Extensive } \\
\text { hepatic } \\
\text { metabolism }\end{array}$ & $\begin{array}{c}\text { Renal } \\
\text { elimination }\end{array}$ \\
\hline \hline Benzodiazepines & 39 & + & ++ & + \\
Doxylamine, diphenhydramine & 9 & - & ++ & + \\
Tricyclic antidepressants & 8 & + & +++ & + \\
Neuroleptics & 6 & + & +++ & + \\
Acetaminophen & 6 & - & + & ++ \\
Aspirin & 2 & + & + & ++ \\
Barbiturates & 2 & + & + to & ++ \\
Betablockers & 1 & + & ++ & + \\
Antiarrhythmics & 0.8 & & & + \\
Others & 25.2 & & & + \\
\hline
\end{tabular}

the latter case, a highly optimized approach that uses sophisticated equipment may be used to detect just a single compound or a well-defined class of compounds. If the objective is to identify an unknown toxic substance that is responsible for acute poisoning under emergency conditions, the demands on the analytical procedure are completely different.

The analytical approach has to cover as many relevant toxic substances as possible. The toxic substance has to be distinguished from endogenous or foodrelated substances and environmental toxicants in the biological matrix, which may be subject to considerable interindividual variation. Because invasive treatment such as hemoperfusion, plasmapheresis, or hyperventilation may be the consequence of the results of a general unknown screening, the analytical toxicologist has a great responsibility for the reliability of his findings. Furthermore, the analytical procedure has to be tested in a large number of acute poisonings with different substances under routine conditions.

A major requirement for general unknown screening is its universal applicability to most classes of toxic substances. However, it is not necessary to demand inclusion of some special substances like ethanol, volatiles, digitalis glycosides, or heavy metals in this universal procedure. In practice, detection of these substances does not pose a major problem because ethanol, digitoxin, or digoxin determination is routinely performed by most hospital laboratories, whereas heavy metal toxicity is typically so specific in its symptom pattern that the diagnosis is hard to miss. Volatiles, which typically cause a specific odor of the exhaled air, may be identified by a modified general unknown screening by gas chromatography-mass spectrometry (GC-MS) by using lower temperatures for the gas chromatography (GC) procedure [6].

GC-MS offers unique advantages over other competing analytical methods for a general unknown screening [3-5, 7-12]. It has been in use for three decades and is highly specific, highly sensitive, universally applicable, covers a large number of relevant toxic substances, and requires relatively little time for an emergency analysis. However, for reasons of cost, it is not possible to provide a 24 -hour service for a general unknown screening by GC-MS in all areas of a country but only in special centers mainly located in urban regions [2]. A rather long transportation time of samples to a toxicological laboratory may be a limiting factor in cases of serious poisoning. If a GC-MS facility is not available, other analytical methods, especially those available in many hospital laboratories, may be considered as a compromise for a general unknown screening. Evaluation of these methods has been enforced and sponsored by a German research program of the Deutsche Forschungsgemeinschaft $[2,6,13-15]$.

Analytical methods that compete with a GC-MS approach to a general unknown screening are combinations of immunoassays, which are commercially available for many classes of drugs like benzodiazepines, opiates, barbiturates, acetaminophen, tricyclic antidepressants, theophylline, and anticonvulsive drugs [1]. These immunoassays are frequently available in hospital laboratories [1]. A major disadvantage of immunoassays is the limited number of drugs that are covered by a combination of the assays. It is desirable that positive results of an immunoassay be confirmed by an independent analytical method [1]. The same is true for general unknown screening procedures that use thin-layer chromatography (TLC), gas chromatography, or high-performance liquid chromatography (HPLC) $[1,16,17]$. The specificity of the latter method may be improved considerably by the use of a diode array detector. However, all these methods lack specificity and universal applicability compared to GC-MS [1]. This disadvantage may be overcome partly by using different solvent and detection systems in TLC or different GC or HPLC columns $[2,6,12,13,16,17]$. 


\section{Types of Samples}

In acute poisoning, gastric content or gastric lavage fluid, blood, plasma, serum, and/or urine may be analyzed. Analysis of gastric content or gastric lavage fluid will provide information on an ingested drug that has not yet undergone complete absorption. Detection of toxicants, drugs, or their metabolites in urine will provide information on substances that have undergone at least partial hepatic metabolism and have already passed through the body [5]. After complete absorption of a toxic substance, the quantification of a toxicant, drug, or their metabolites in blood, plasma, or serum allows an estimation of the severity of an acute intoxication (see Table 2).

For practical purposes and restriction of costs, a screening procedure should focus primarily on urine, which contains higher concentrations of most drugs and toxicants than blood, serum, or plasma. However, it has to be kept in mind that many substances undergo nearly complete metabolism, which means that in overdose with substances subjected to extensive hepatic metabolism, only metabolites but not the parent compound may be detectable in urine. Because the dose range in drug or toxicant overdose is typically several milligrams to grams, sensitivity is not a special problem in urine analysis. Urine concentrations typically vary between the nanogram to microgram per milliliter range. Unexpectedly high concentrations of a toxic substance and its metabolites in urine may pose some problems with GC column overload and memory effects in the ion source of the mass spectrometer.

With few exceptions, most drug or toxicant metabolites have a considerably higher polarity than their parent compounds, which may render the extraction yield achieved with organic solvents rather poor, especially if conjugates are formed. This problem can be overcome by conjugate cleavage by hydrochloric acid hydrolysis or enzymatic hydrolysis with glucuronidase-sulfatase [5]. For an emergency procedure, hydrochloric acid hydrolysis requires considerably less time than enzymatic cleavage. However, the former method is frequently associated with extensive artifact formation [5]. Because conjugate formation is frequently incomplete, sufficient amounts of unconju- gated toxic subtances or their metabolites are still present in urine for extraction and identification. Because highly polar metabolites frequently do not pass the gas chromatograph or may be prone to thermal decomposition, simple derivatization procedures are required [5].

In patients with anuria or acute renal failure, blood, plasma, or serum have to be used for the general unknown screening. The concentration range of a toxic substance in blood, plasma, or serum is typically lower compared to urine. However, the relative fraction of the unchanged toxic substance to its metabolites is frequently higher in blood compared to urine, which may facilitate the identification process. For a GC or GC-MS screening of volatiles, blood, plasma, or serum is generally the specimen of choice [6].

\section{Extraction and Derivatization}

For practical reasons, liquid-liquid extraction and solid-phase extraction are the most common extraction procedures in emergency toxicology. Liquid-liquid extraction frequently is preferred because it is more universally applicable to many classes of toxic substances [5]. A variety of columns for solid-phase extraction are commercially available. Because an ingested drug or toxicant may be acidic, neutral, or basic, extraction should be done at $\mathrm{pHs}$ of 3-4 and 8-9. For simplification both extracts may be unified. As a further step toward reducing the time for emergency analysis, the $\mathrm{pH}$ may be kept at about 8-9. Under this condition, most relevant drugs and toxicants and even acidic compounds with few exceptions (e.g., salicylic acid) will be extracted in sufficient quantities to be detected by the GC-MS procedure [3].

The most frequently used solvents for extraction are ethylacetate, diethylether, or mixtures of methylene chloride-isopropanol $[4,5]$. The organic phase is evaporated to dryness and the residue frequently is dissolved in methanol $[4,5]$. If the latter is used, one has to keep in mind the possibility of artifact formation.

Due to the high polarity and thermal instability of many drugs, toxicants, and especially their metabolites, derivatization is advisable. Derivatization should preferrably be performed by using techniques that are

Table 2. Information derived from analysis of different specimens for a general unknown screening in acute poisoning

\begin{tabular}{lc}
\hline Specimen & Information provided by GC-MS analysis \\
\hline \hline Gastric lavage fluid/gastric content & $\begin{array}{c}\text { Ingested drugs and toxicants that have not } \\
\text { yet been absorbed } \\
\text { Ingested drugs and toxicants that have } \\
\text { undergone absorption, metabolism, and renal } \\
\text { elimination } \\
\text { Blood/plasma/serum }\end{array}$ \\
& $\begin{array}{l}\text { Ingested drugs and toxicants that have } \\
\text { undergone absorption and to some extent } \\
\text { metabolism }\end{array}$ \\
\hline
\end{tabular}


fast and have a high yield of derivatives. For practical reasons, methylation that uses diazomethane or methyl iodide-potassium carbonate, acetylation with acetic anhydride, trifluoroacetylation with trifluoroacetic anhydride, and pentafluoropropionylation with pentafluoropropionic anhydride have some advantages over trimethylsilylation or use of organoboronic acid with respect to GC column bleeding and memory effects in the ion source of the mass spectrometer $[3,5,18]$. However, it has to be kept in mind that derivatization may be associated with considerable artifact formation [3].

\section{Gas Chromatography}

Capillary columns are used as the standard in GC-MS screening procedures. Commercially available capillary columns allow adequate separation of the complex mixtures typically present in urine extracts. A rapid temperature program is used to limit the duration of the GC run, which typically ranges from 15-25 min. The maximum GC column temperature is typically in the range of $300^{\circ} \mathrm{C}$.

\section{Mass-Spectral Techniques}

Due to the special requirements of a general unknown screening, electron impact (EI) is still the most widely applied mode of ionization [3-5]. It has the advantage that it generates mass spectra with sufficient fragments to allow identification by a search of large EI mass spectra libraries. Other ionization techniques like positive or negative chemical ionization, atmospheric pressure ionization, electrospray ionization, thermospray ionization, fast-atom bombardment, or the directly exposed probe in combination with tandem mass spectrometry techniques may be useful additional options that provide complementary information, for example, on the molecular ion, to identify an unknown drug or toxicant [19-22].

Secondary fragment ion mass spectra may be obtained from molecular ions or $[\mathrm{M}+1]^{+}$ions by collisioninduced dissociation. However, these secondary mass spectra may differ considerably from electron impact spectra, which renders a library search with an EI database difficult and limited in its reliability. Therefore, the standard ionization mode for a general unknown screening is still electron impact ionization.

\section{Identification of Drugs and Toxicants, their Metabolites, Derivatives, and Artifacts, Endogenous Substances, Food-Derived Substances, and Environmental Contaminants}

In urine extracts, unchanged drugs, toxicants, their metabolites, artifacts, and derivatives as well as endogenous substances and environmental contaminants may be present. Furthermore, substances derived from food or cigarette smoke like caffeine, theobromine, benzoic acid, hippuric acid, nicotine, and cotinine may be found [5]. Typical environmental contaminants include phthalic esters, hydroxylated biphenyls, DDT and its metabolites, and pentachlorophenol [5]. Therapeutic drugs frequently identified in urine extracts are lidocaine and catecholamines. Sulfur $\left(S_{8}\right)$ may be an artifact from therapeutic administration of high doses of $\mathrm{N}$-acetylcysteine used in acetaminophen overdose. A urine extract typically contains abundant peaks of cholesterol, androstanol derivatives, fatty acids, benzoic acid, hippuric acid, phenols, and theobromine [4].

A problem in the identification of an unknown agent may be coelution with other substances and/or peak tailing, which requires a background subtraction by an appropriate algorithm. Dependent on the subtraction algorithm and the subtraction modalities chosen by the operator, the resultant mass spectra may be subject to considerable alterations in fragment ion abundances. This may render identification of an unknown by a mass spectra library search rather difficult and prone to false interpretations.

For identification of unknown agents, an automated mass spectra library comparison by computer is mandatory. However, large commercially available libraries frequently do not contain all metabolites, artifacts, and derivatives typically found in urine extracts. An updated data file especially for a general unknown GC-MS screening has been published by Pfleger, Maurer, and Weber [5].

In our experience, a general unknown screening of urine identifies previously unknown metabolites of a drug or toxicant in up to $10 \%$ of all cases of intoxications with rare substances [4]. One reason is that drug or chemical overdose is associated with comparatively high concentrations of metabolites in the urine, which facilitates the identification of metabolites that are formed only in trace amounts under therapeutic dosing conditions or normal exposure. A large number of previously unknown metabolites, artifacts, and derivatives of drugs and toxicants detected by a general unknown screening in acute poisonings have been published. For previously unknown drug metabolites detected in drug overdose, further investigation gave evidence that all metabolites also were formed under a therapeutic dosing regimen (e.g., tiaprofenic acid, amantadine, tromantadine, dextromethorphan, chlorphenoxamine, ajmaline, melperone, imipramine, pipamperone, chloroquine, propallylonal, and kavaine) [23-34].

The thermal challenge of the GC procedure may lead to artifact formation. Dehydration and formation of olefins is rather common [5]. In addition, the heat challenge may lead to ring formation and dehydration whenever a thermodynamically stable ring can be formed [5]. Artifact formation further may be due to $\mathrm{N}$-oxidation of tertiary amines when diethylether is 
used as a solvent, which may contain traces of peroxide [5]. Similarly, sulfur in phenothiazine may be oxidized to sulfoxides [5]. The thermal challenge of GC may lead to decarboxylation of carboxylic acids [5]. $\mathrm{N}$-oxides may undergo Cope elimination [5]. Derivatization by methylation or acetylation will lead to the formation of the corresponding methyl and acetyl derivatives. Derivatization with acetic anhydride is frequently associated with dehydration of alcohols [5]. Sometimes the use of methanol for reconstitution of an evaporated extract may lead to the formation of methylesters from carboxylic acid [5]. Furthermore, methanol may thermally decompose to traces of formaldehyde in the injection port of the gas chromatographer, which then may undergo condensation with primary or secondary amines or induce ring closure of 1,2-aminoalcohol groups, which are present, for example, in flecainide and most betablockers (e.g., metoprolol, see Figure 1) [35].

Furthermore, artifacts may be formed during hydrochloric acid hydrolysis, which may lead to cleavage of an ether bridge in betablockers or alkanolamine antihistaminic drugs. Under hydrochloric acid hydrolysis, many 1,4-benzodiazepines are cleaved to form aminobenzophenone derivatives [5].

\section{Identification of Toxic Substances}

A careful approach to background subtraction, deconvolution of overlapping peaks is a major prerequisite for identification of unknown agents by a library search. It requires thorough training and great experience in the field of mass spectrometry as well as clinical toxicology. In a first approach, physiological endogenous substances and substances not related to acute poisoning have to be distinguished from exogenous toxic substances. Furthermore, food ingredients and typical environmental contaminants have to be identified. Then the drugs or toxicants that cause the acute poisoning as well as their metabolites, derivatives, and artifacts have to be identified. A mass spectra library, which should contain a sufficient number of relevant reference spectra, is mandatory. Due to extensive metabolism or artifact formation, many drugs or toxicants can be detected only indirectly, for instance, by identification of their corresponding metabolites, artifacts, or derivatives. In such cases, it must be kept in mind that an artifact, metabolite, or derivative sometimes may be generated from different precursors.

The interpretation process in a general unknown screening by GC-MS may become rather time-consuming if a drug is metabolized to a large number of metabolites as, for instance, propyphenazone, which forms about 20 metabolites [5]. Interpretation is further complicated if more than one drug is ingested, which is typically the case in about $46 \%$ of all suicidal cases of drug overdose [4].

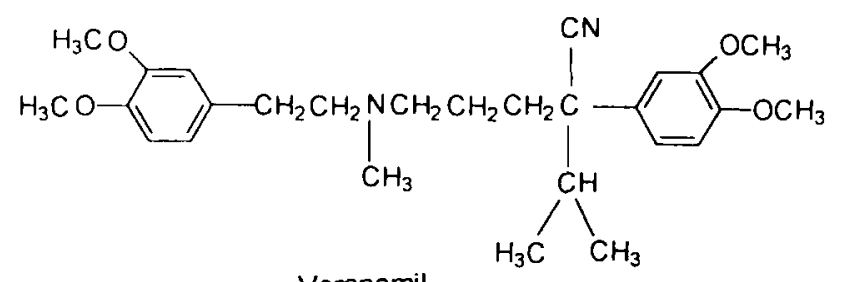

Verapamil
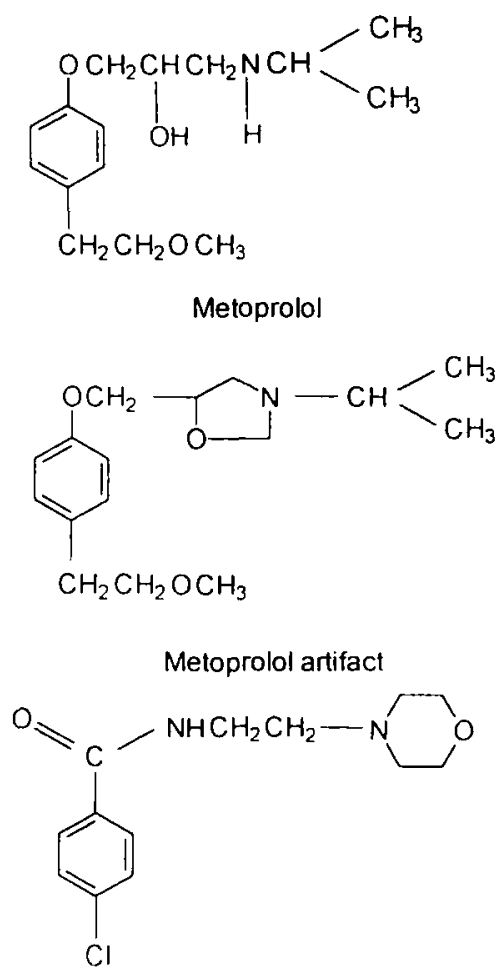

Moclobemide

Figure 1. Verapamil, metoprolol, metoprolol artifact, and moclobemide.

The results of the general unknown screening are required by the treating physician in emergency wards as rapidly as possible. A general unknown screening procedure takes an average of $2 \mathrm{~h}$ [4]. Subsequent quantification of the identified toxic substance in blood, plasma, or serum is necessary to allow an estimation of the severity of the intoxication [1]. Quantification of an identified drug or toxicant should be performed with the most appropriate method of quantification, which may be high-performance liquid chromatography, gas chromatography, or, if available, immunological assays [1]. It need not necessarily be done by massspectral techniques.

The mass spectra library should be updated with all metabolites, artifacts, and derivatives identified by the procedure. The database in this field is still growing at a rapid pace and many previously unknown metabolites and artifacts still have to be identified. 


\section{Examples for the Importance of a Gas Chromatography-Mass Spectrometry Screening Procedure for the Diagnosis of Acute Poisoning}

To exemplify the importance of a general unknown screening by using GC-MS and for a demonstration of the practical problems that may be encountered in the identification and interpretation process, five case reports are presented.

\section{Case 1 (16-Year-Old Fenale)}

A 16-year-old female with a history of school problems was found comatose by her parents. The emergency ambulance staff found the patient in cardiovascular arrest. The parents suspected an intoxication with the calcium antagonist verapamil, which had been prescribed to the mother. Resuscitation was started by the emergency physician and his team with extreme doses of catecholamines. However, because efforts on the site were frustraneous, the patient was transported to the intensive care unit of our hospital with ongoing resuscitation in the emergency ambulance. In the intensive care unit, the patient's circulation could be stabilized.

A general unknown screening gave evidence of the ingestion of the betablocker metoprolol and the monoamino oxidase inhibitor moclobemide, but no verapamil (see Figures 1 and 2 ). In addition to unchanged moclobemide and metoprolol, hydroxymetoprolol and an artifact generated by reaction of formaldehyde with metoprolol could be identified [35]. Artifact formation was due to the use of methanol as the solvent for the extract. Trace amounts of formaldehyde are formed by thermal decomposition of methanol during the GC procedure.

In this case, the identification of metoprolol and moclobemide rather than verapamil in urine by the general unknown screening was very important for

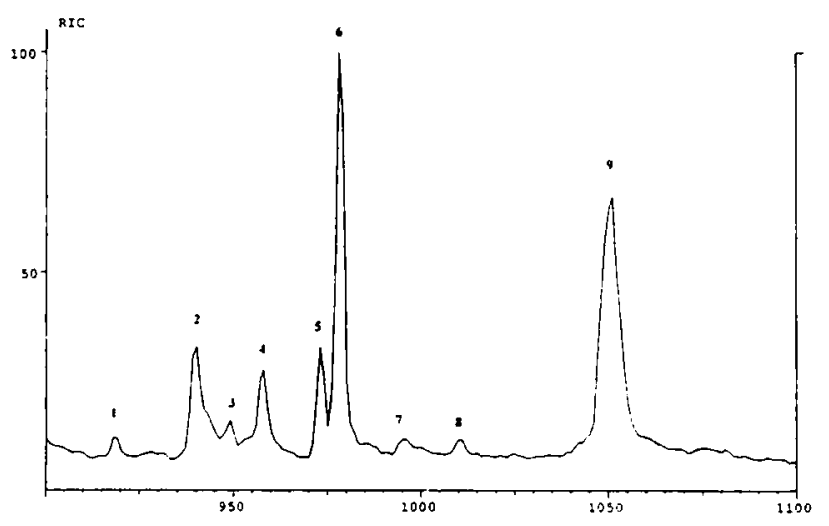

Figure 2. Part of the total ion chromatogram of the urine extract of case $1(1=$ heptadecanoic acid methyl ester, 2 = metoprolol, $3=$ acetic acid octadecyl ester, $4=$ octadecenoic acid methyl ester, $5=$ octadecanoic acid methyl ester, $6=$ metoprolol artifact, $7=$ ethyl phenoxy benzene, $8=$ docasane, $9=$ moclobemide). therapy because verapamil poisoning refractory to standard treatment might have been by intravenous administration of high doses of calcium gluconate. This type of treatment occasionally has been reported to be successful in severe poisoning, although controlled studies are not available. However, high-dose calcium in calcium antagonist poisoning may be associated with the risk of a decrease in cerebral perfusion and cerebral ischemia.

Circulation was stabilized further by high-dose infusion of catecholamines and high doses of glucagon. The patient recovered completely and was discharged without neuropsychological sequelae. In this case, the information provided by the parents about the probable ingested drug was not correct. The results of the general unknown screening had important implications for the further therapeutic management of the patient.

\section{Case 2 (47-Year-Old Male)}

A 47-year-old technician was found with drowsiness and a greyish discoloration of the skin in his laboratory by colleagues. He stated that, in a suicide attempt, he had ingested a chemical from a can, which was labeled "dimethylsulfone." The patient was admitted to the intensive care unit of the hospital. The greyish discoloration of the skin was due to $65 \%$ methemoglobinemia in the blood, as determined by blood gas analysis. Therapy included administration of ascorbic acid and toluidine blue to enhance the reduction of methemoglobin to hemoglobin. However, very little is known about dimethylsulfone toxicity, especially in humans. From a theoretical point of view, it seems to be rather unlikely that dimethylsulfone is a significant oxidant to cause methemoglobinemia.

A toxicological screening of urine extracts by GC-MS was performed. Dapsone, but no dimethylsulfone, could be identified (Figure 3). Dapsone is an antibiotic and immunomodulator used for treatment of leprosy and Duhring's disease. It is structurally related to sulfonamides. This class of drugs is known to cause

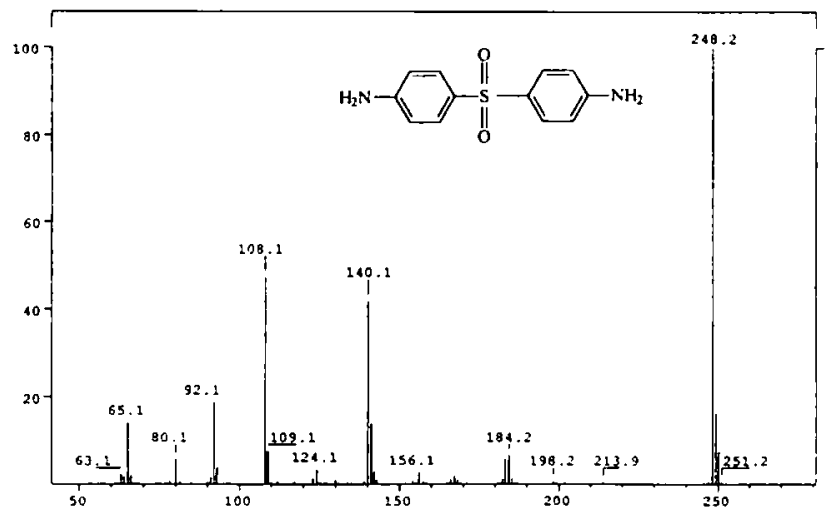

Figure 3. Electron impact mass spectrum of dapsone. 
methemoglobinemia. The patient fully recovered and was discharged in complete health.

In this case, GC-MS helped to identify the drug that caused the methemoglobinemia, although no hints or circumstances of the case had pointed to this type of chemical. The label on the can obviously had not been correct.

\section{Case 3 (76-Year-Old Fenale)}

A 76-year-old female was found comatose in her apartment by her neighbors. The reason for coma remained unclear. There were no empty drug containers, which might have suggested suicidal drug overdose. The patient was admitted to the ICU of the hospital. Respiratory insufficiency necessitated ventilator therapy. A toxicological screening performed with the patient's urine used immunological tests for benzodiazepines, barbiturates, opiates, tricyclic antidepressants, and acetaminophen. All toxicological tests were negative and poisoning seemed to be very unlikely. Cerebral bleeding, apoplexy, and basilar thrombosis were ruled out by cerebral computed tomography and digital subtraction angiography. Laboratory tests gave no evidence for a metabolic dysregulation, which might have caused the coma. The cerebrospinal fluid was normal. Because the patient remained in a coma for the next two days and an EEG showed burst suppression (intermittent isoelectric signals for $10 \mathrm{~s}$ ), the toxicological screening was repeated, now via a GC-MS procedure. Glutethimide was identified (Figure 4). Plasma level data indicated severe glutethimide overdose. Glutethimide is a barbituratelike hypnotic that was used in Germany until the 1970 s and then disappeared from the market. Obviously, this patient had stored containers with this drug in her apartment for a very long time and then used it to attempt suicide.

Detoxification by repeated administration of activated charcoal via a nasogastric tube and forced diuresis was initiated. The patient's alertness gradually improved and she was discharged in complete health after two weeks.

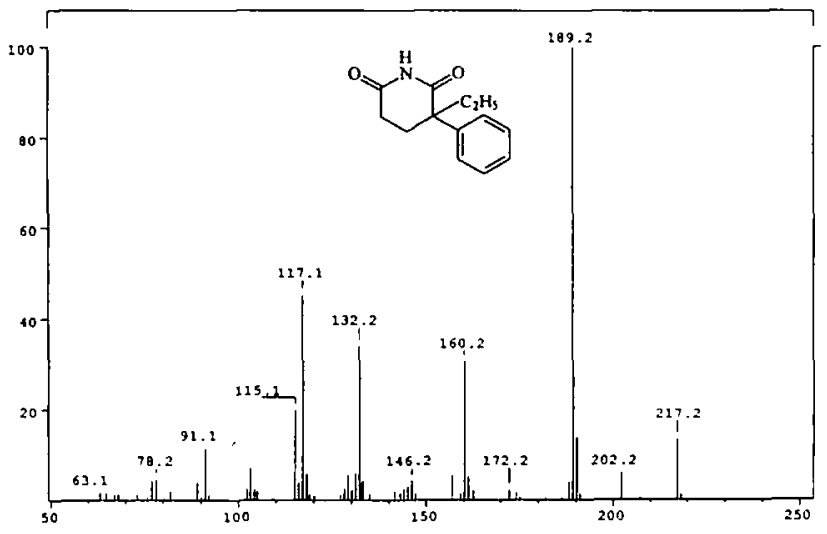

Figure 4. Electron impact mass spectrum of glutethimide.
This case demonstrates that a GC-MS screening is superior to a screening via immunological methods, which only cover a limited number of drugs.

\section{Case 4 (72-Year-Old Female)}

A 72-year-old female with no history of previous epilepsy was found with grand-mal seizures in her apartment by her neighbors. On admission to the hospital, the patient presented with a generalized epileptic state and coma. A neurological disease was assumed because no empty drug containers suggested drug overdose. A toxicological screening by immunological methods that covered barbiturates, opiates, tricyclic antidepressants, stimulants, acetaminophen, theophylline, and carbamazepine was negative. A thorough diagnostic procedure gave no evidence for neurological or metabolic disorders. Because manifestation of primary grand-mal seizures seemed to be very unlikely at an age of 72 years, the toxicological screening was repeated via a GC-MS procedure. Methaqualone and four of its metabolites were identified in urine (Figure 5). Methaqualone overdose is extremely rare because the drug disappeared from the market in 1980 after it became subject to narcotic prescription law. The clinical characteristics of methaqualone overdose are hyperexcitability, seizures, and coma.

This case again exemplifies the superiority of a GC-MS screening procedure over other methods, especially immunological techniques.

\section{Case 5 (16-Month-Old Female)}

A 16-month-old infant was found cyanotic in her bed by her parents. On arrival of the emergency physician, the patient had cardiorespiratory arrest due to an unknown cause. Resuscitation was started. A general unknown screening via GC-MS yielded artifact $A$, but not $B$, which are typically formed after injection of thermally instable ergotamine into the GC-MS (Figure 6).

Further resuscitation efforts were frustraneous. Poisoning of the child with ergotamine was assumed and a medicolegal investigation was initiated. The samples

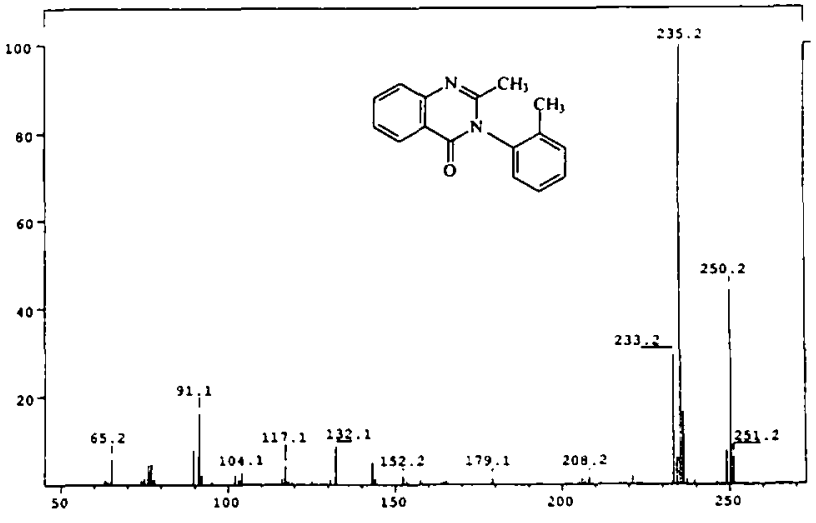

Figure 5. Electron impact mass spectrum of methaqualone. 
<smiles>CC1C(=O)N2CCCC2C2(O)OC(C)(NC(=O)C3C=C4c5cccc6[nH]cc(c56)CC43)C(=O)N12</smiles>

Ergotamine

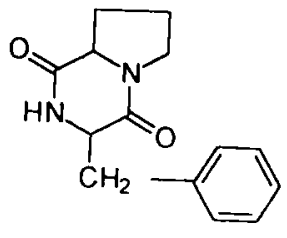

A

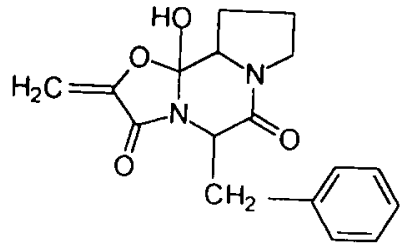

$B$
Figure 6. Major thermal decomposition products $A$ and $B$ (artifacts).

were sent to our laboratory for confirmation as well as determination of ergotamine by HPLC. However, no ergotamine could be demonstrated in the blood extract at a detection limit of $0.6 \mathrm{ng} / \mathrm{mL}$ (toxic concentration range $>3 \mathrm{ng} / \mathrm{mL}$ ). Mass-spectral analysis was performed by using the direct exposed probe in the negative ionization mode, which yielded two characteristic

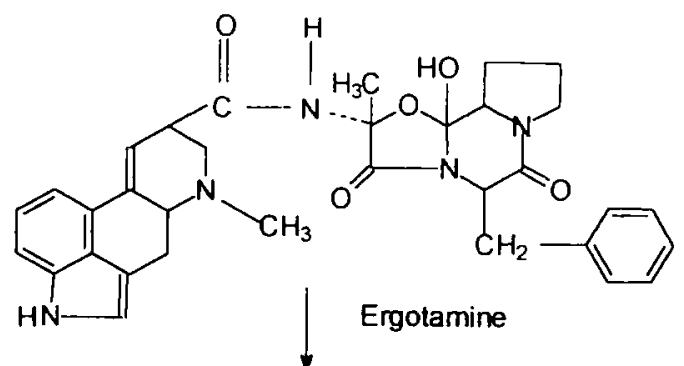

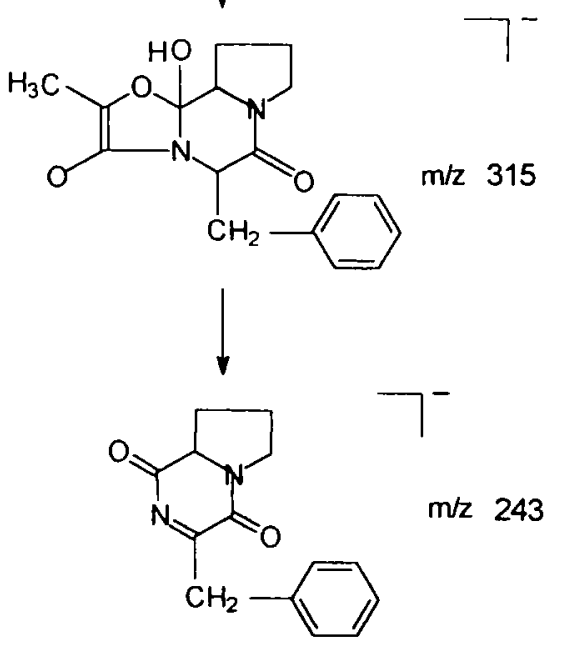

Figure 7. Major fragment ions of ergotamine in the negative chemical ionization mode with methane. fragment ions (Figure 7) [36]. HPLC analysis with fluorescence detection was likewise negative $(<1$ $\mathrm{ng} / \mathrm{mL}$ ) for ergotamine. However, the presence of artifact $A$ but not $B$ (Figure 6) could be confirmed by GC-MS. The most likely explanation for detection of A was that this artifact was formed by condensation of phenylalanine with proline, which are both physiologically present in biological fluids (Figure 8 ). The diagnosis in this case was not ergotamine intoxication, but most likely a sudden infant death syndrome, which is multifactorial in its causation according to present knowledge.

If the original substance is too polar and thermally instable to pass the GC in the routine general unknown screening and only artifacts are formed, the interpretation process has to consider that sometimes different parent compounds may be the precursors of an artifact. In this case, no relevant ergotamine concentrations could be detected via special mass-spectral techniques and HPLC. Artifact B could not be detected. The most likely source of artifact $\mathrm{A}$ was condensation of phenylalanine with proline.

\section{Advantages and Disadvantages of a General Unknown Screening Via Gas Chromatography-Mass Spectrometry}

Although gas chromatography-mass spectrometry has become a rather old-fashioned technique, there are currently few alternatives that might be as efficient in identification of toxic substances in acute poisoning. However, GC-MS has some major disadvantages in a general unknown screening procedure:

1. It requires extraction and sometimes conjugate cleavage prior to extraction.

2. Only thermally stable drugs or toxicants can be detected in their undecomposed form by GC-MS. For thermally unstable compounds, derivatization procedures are required.

3. The formation of artifacts from drugs, toxicants or their metabolites, and derivatives complicates the identification process. Daily routine work in this field indicates that many artifacts still remain to be identified $[4,5]$.

The standard ionization mode in a general unknown screening is electron impact ionization. Because reliable identification of toxic substances from an ex-

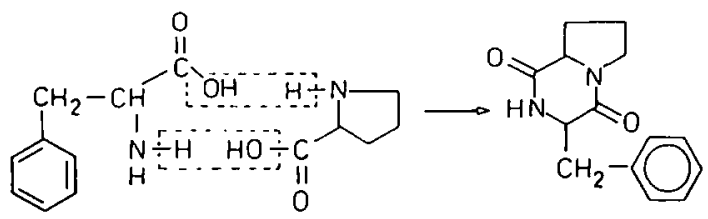

Figure 8. Formation of A by condensation of phenylalanine with proline. 
tract is required, a molecular ion or a $[\mathrm{M}+1]^{+}$ion with no fragment ions and the retention time are not specific enough for a highly reliable identification of an unknown. High reproducibility of a fragment ion mass spectrum is required to make identification by comparison of spectra with an EI library most reliable. The comparability of mass spectra generated from molecular ions by collision-induced dissociation with that of an EI mass spectra library is lower than that of an EI spectrum.

A step in the future might be the routine use of high-performance liquid chromatography-mass spectrometry (HPLC-MS) with atmospheric pressure ionization or use of an electrospray for a general unknown screening. However, several problems have to be solved due to the specific needs of a general unknown screening:

1. Universal HPLC columns or combinations of HPLC columns have to be used.

2. The ionization technique has to be applicable universally and has to provide sufficient sensitivity for a wide range of relevant substances.

3. Furthermore, a fragment ion mass spectrum has to be generated, which should be as comparable as possible with an EI spectrum to enable use of the huge databases of EI mass spectra.

4. The HPLC-MS screening procedure has to be tested under routine conditions in a large number of poisonings with different classes of toxic substances.

5. The mass spectra database has to be completed for drug and toxicant metabolites, artifacts, and derivatives detectable by HPLC-MS.

\section{Acknowledgment}

We are indebted to Professor Dr. F. W. McLafferty, Department of Chemistry, Cornell University, Ithaca, NY, for many stimulating discussions.

\section{References}

1. Deutsche Forschungsgemeinschaft (DFG), Einfich twrikologische Laboratoriumsuntersudungen bo akuten Virgiftungen (Senatskommission für Klinisch-toxikologische Analytik, Mitteilung XXIII); VCH: Weinheim, 1995.

2. Geldmacher von Mallinckrodt, M. Klimisch-toxikologische Amalutik, gegenswärtiger Stand und Forschumg fïr dic Zukumft; VCH: Weinheim 1983; pp 22, 43.

3. Köppel, C.; Tenczer, J. Int. I. Mass Spectrom. Lon Phys. 1983, 48, 213-216

4. Köppel, C., Tenczer, J. Intensiumet. 1988, 25, 289-293.

5. Pfleger, K.; Maurer, H. H.; Weber, A. Mass Spectral and CC DATA of Drugs, Poisons, Pesticides, Pollutants and Their Metabolites, Vol, I; VCH: Weinheim, 1992.

6. Deutsche Forschungsgemeinschaft (DFG), Gas Chromatographic Retention Intices of Solients and Other Volatile Suldstances for Usc in Toxicological Analysis (Senatskommission für
Klinisch-toxikologische Analytik. Report XIX, Special Issue of the TIAFT Bulletin); VCH: Weinheim, 1992.

7. Finkle, B. S.; Taylor, D. M.; Bonelli, E. J. J. Chromatogr. Sci. 1972, 10, 312-333.

8. Finkle, B. S.; Foltz, L.; Taylor, D. M. /. Chromatogr. Sci. 1974, 12, 304-336.

9. Horio, T.; Loeffler, K. Clin. Chim. Acta 1973, 49, 445-454.

10. Law, N. C.; Aandahl, V.; Fales, H. M.; Milne, G. W. A. Clin. Chim. Acta 1971, 32, 221-228.

11. Oswald, E. O.; Albro, P. W.; McKinney, J. D. J. Chromatogr. 1974, 98, 363-448.

12. Deutsch, D. G.; Bergert, R. J. Clin. Chem. 1985, 31, 741-746.

13. Deutsche Forschungsgemeinschaft (DFG), Thin-Lnyer Chromatographic $R_{f}$-Value's of Toxicologically Releciant Substances on Standardized Systems (Senatskommission für Klinisch-toxikologische Analytik. Report XVII, Special Issue of the TIAFT Bulletin); VCH: Weinheim, 1992.

14. Deutsche Forschungsgemeinschaft (DFG), Gas Chromatosraphic Retention Indices of Toxicologically Relevant Substances on Packed or Capillary Columns with Dimethylsilicone Stationary Phase's (Senatskommission für Klinisch-toxikologische Analytik. Report XVIII, Special Issue of the TIAFT Bulletin); VCH: Weinheim, 1992.

15. de Zeeuw, R. A.; Franke, J. P.; Maurer, H. H.; Pfleger, K. Gas-Chromatographic Retention Indices of Toxicologically Relevant Substances and Their Metabolites (Report of the DFG Commission for Clinical-Toxicological Analysis, Special Issue of the TIAFT Bulletin), 3rd ed.; VCH: Weinheim, 1971.

16. Binder, S. R.; Regalia, M.; Biaggi-McEachern, M.; Mazhar, M. 1. Cirromatogr. 1989, 473, 325-341.

17. de Zeeuw, R. A.; Hartstra, J.; Franke, J. P. I. Chromatogr. 1994, 674, 3-13.

18. Maurer, H. H. I. Chromatogr. 1990, 530, 307-326.

19. Covey, T. R.; Lee, E. D.; Henion, J. D. Anal. Chem. 1986, 58 , 2453.

20. Lee, E. D.; Covey, T. R.; Muck, W.; Henion, J. D. I. Clromatogr. 1988, 458, 313-321.

21. Jehuda, Y. For'nsic Sci. Prog. 1991, 5, 1-29.

22. Henion, J. D. In Mass Spectromctry in Biomedical Research; Gaskell, S., Ed.; Wiley, New York, 1986.

23. Köppel, C.; Tenczer, J. Arzmim. Forsch./Drug Res. 1984, 34, 836-837.

24. Köppel, C.; Tenczer, J. Arnemim. Forsch./Drug Res. 1985, 35, $1334-1335$.

25. Köppel, C.; Tenczer, J. Bioned. Mass Spectrom. 1985, 12, 497-498.

26. Köppel, C.; Tenczer, J. Biomed. Mass Spectrom. 1985, 12, $499-501$.

27. Köppel, C.; Tenczer, J. Arzmim. Forsch./Drug Re's. 1987, 37, $1062-1064$.

28. Köppel, C.; Tenczer, J. Arzn'im. Forsch./Drug Re's. 1987, 37, $208-211$.

29. Köppel, C.; Tenczer, J. Arznim. Forsch./Drug Re's. 1987, 37, 1304-1306.

30. Köppel, C.; Tenczer, J. I. Chrumatogrt. 1988, 427, 144-150.

31. Köppel, C.; Tenczer, J. I. Chromatogr. 1988, 431, 197-202.

32. Köppel, C.; Tenczer, J. I. Chromatogr. 1989, 491, 432-438.

33. Köppel, C.; Tenczer, J. Eur. 1. Drug Metab. Pharmacokinet. 1989, 1t, 309-316.

34. Duttfield, A. M.; Jamieson, D. D.; Lidgard, R. O.; Duffield, P. H.; Bourne, D. J. I. Chromutogr, 1989, 475, 273-281.

35. Köppel, C.; Tenczer, J.; Peixoto-Menezes, K. M. I. Clromatogrr. 1991, 563, 73-81.

36. Haering, N.; Settlage, J. A.; Sanders, S. B.; Schubert, R. Biomed. Mass Spectrom. 1985, 12, 197-199. 